\title{
A burning desire to understand genetic mutations in gastroenterology
}

\author{
Paul C Adams MD ${ }^{1}$, Editor-in-Chief, Peter Ainsworth MB ChB PhD²
}

$\mathrm{D}_{\mathrm{s}}^{\mathrm{r}}$ Peter Ainsworth is an Adjunct Professor in the Department of Biochemistry at the University of Western Ontario, London, Ontario. He is the Director of the Molecular Diagnostics Laboratory of the London Laboratory Services Group, which provides diagnostic genetic testing for Southwestern Ontario.

The Human Genome Project has greatly expanded our understanding of the pathogenesis of gastrointestinal diseases. However, the function of many identified genes remains unknown. Molecular genetics is a relatively new scientific field, and thus, many of its fundamental concepts would not have been part of a gastroenterologist's education. In the present review, an analogy is made between genetic mutations and digital music copying to increase our comprehension of this complex field.

In 2003, the Human Genome Project announced their results from a 13-year multinational study which identified approximately 30,000 genes in human DNA. This achievement represents a landmark in modern medicine; however, the field of molecular genetics can be bewildering to the practicing clinician, who may only see an array of alphabet soup and overly complex scientific articles. Simply put, a genome represents all of the nuclear DNA in an organism, including the coding sequences carried in the genes. Genes carry information for making proteins that are involved in cell function. DNA is much like a double-stranded beaded necklace made up of four bases, strung out in pairs (guanine-cytosine [G-C] and adenine-thymine [A-T]) along two interwoven strands of a sugar-phosphate polymer backbone, to make up a double helix comprising the genome of an organism. Fully extended, the DNA in humans is approximately $2 \mathrm{~m}$ in length and contains approximately $3.5 \times 10^{9}$ base pairs.

The use of digital computer technology to record and copy music is also a complex scientific field. Although the compact disc (CD) was only introduced in 1983 by Royal Philips Electronics Ltd (The Netherlands), the pulse code modulation technology used on CDs was developed in 1937 by British scientist Alec Reeves. The concept for the CD was developed in 1969 by a Dutch physicist, Klaas Compaan, and an American scientist, James T Russell. The first recordable CD, which led to the copying of CDs, was released in 1988. Today, millions of music and video discs have been sold and copied, and the entertainment industry is convinced that this has led to a major decline in their sales and profits. The lessons learned from music copying are used as an analogy in the present article for the study of genetic mutations associated with gastroenterological diseases.

In this analogy, we will assume that our genetic code is the digital information on the original disc and during the process of copying, genetic mutations have developed. When the disc was introduced, it was assumed that the copying of the digital information would be 'perfect'; however, it is now well known that many of the copied CDs do not contain exactly the same information as the original. This may be related to burning speed, compression of digital data, copy protection methods, quality of the copier and disc, as well as the algorithm for the data transfer.

\section{MUTATION TYPES \\ Germline mutation}

A defect on the original disc will reappear on every copy in an analogous fashion to the transmission of a germline mutation which will be represented in every cell (Table 1).

\section{Somatic mutation}

A defect that develops spontaneously in one of the CD copies will not be present in the other copies, which is similiar to a somatic mutation in human DNA found, for example, in a liver tumour (eg, in the p53 gene), but would not likely be present in the cells of other tissue types.

\section{Point mutations}

The story of evolution continues to generate controversy for individuals who hold more fundamentalist beliefs, especially regarding observations flowing from the Human Genome Project establishing the remarkable similarity of the insect, rodent and human genome.

The vast majority of point mutations in the human genome are $\mathrm{C}>\mathrm{T}(\mathrm{G}>\mathrm{A})$ nucleotide substitutions resulting from the relative instability of methylated cytosine residues in $\mathrm{CpG}$ dinucleotides. These mutations arise by chance mutation events but may be propagated by some less obvious heterozygote advantage. A pristine $\mathrm{CD}$ that has been scratched after lying on the floor without a case could have a damaged code resulting in a skip in the music during playback, analogous to a genetic deletion event (mutation) interrupting the correct transfer of information from genetic code to messenger RNA to protein, a mutation that greatly alters the protein and frequently results in clinical disease. The DNA in every cell is continuously exposed to injury by various intrinsic and extrinsic factors. Usually, the consequences of damage to the DNA

\footnotetext{
${ }^{1}$ Department of Medicine, London Health Sciences Centre; ${ }^{2}$ Department of Biochemistry, University of Western Ontario, London, Ontario Correspondence: Dr Paul C Adams, London Health Sciences Centre, 339 Windermere Road, London, Ontario N6A 5A5.

Telephone 519-685-8500 ext 35375, fax 519-663-3549, e-mail padams@uwo.ca
} 
TABLE 1

An analogy between human genetic mutations and digital music copying

\begin{tabular}{|c|c|}
\hline Mutations & Compact discs \\
\hline Germline & Present on the original disc and all subsequent copies \\
\hline Somatic & Present on the copy but not on the original disc \\
\hline \multicolumn{2}{|l|}{ Point } \\
\hline Missense & $\begin{array}{l}\text { A digital ' } 0 \text { ' is substituted with a ' } 1 \text { ' and a music error } \\
\text { may or may not occur }\end{array}$ \\
\hline Nonsense & $\begin{array}{l}\text { An error occurs in the lead-in sequence and the whole } \\
\text { disc will not play }\end{array}$ \\
\hline
\end{tabular}

are not severe, because the molecule is subject to various surveillance and repair systems that minimize the occurrence of mutations. These DNA damage control pathways may lead to either repair of the errors or programmed cell death. DNA repair systems can be separated into several discrete groups, including nucleotide excision repair, DNA double-strand break repair or DNA mismatch repair (MMR). In the latter example of defective DNA repair, loss of function of one of the genes responsible for MMR on human chromosome 2 or 3 can lead to hereditary nonpolyposis colorectal cancer syndrome, resulting from failure to recognize and repair the base pair mismatches or small insertion/deletion mutations that may occur during DNA replication. In many $\mathrm{CD}$ players, an error correction circuit allows for the smooth playback of damaged discs in a manner similar to MMR in genes. This concept may also occur in humans in the successful translation of proteins from defective genetic material, and may be important in inhibiting the development of neoplasia. In an analogous fashion, newer methods of CD copy protection can include the intentional inclusion of nonsense information to overwhelm the error correction circuitry to block the copying process.

Missense mutation: A common genetic defect is the substitution of one base pair by another base pair - a missense mutation - which can lead to the production of a defective protein. Similarly, on a music disc, the digital information may not be copied properly and a ' 0 ' may be substituted for a ' 1 ' in the stream of digital information. In humans, an example of a common point mutation may be found in hereditary hemochromatosis, in which a nucleotide substitution $845 \mathrm{G}>\mathrm{A}$ leads to the replacement of the normal cysteine in the HFE gene by the amino acid tyrosine, which subsequently alters the function of the protein and can lead to iron overload. A common observation is that a copied disc may play well in a home player but may skip in the car player, which can serve to illustrate the concept of variable clinical expression or incomplete penetrance of defects, a phenomenon which is commonly seen in hemochromatosis.

Nonsense mutation: All human proteins begin with the same specific codon (ATG) coding for the amino acid methionine. The signal for termination of copying of a gene is effected by a specific termination or 'stop' codon (TAA, TAG or TGA) that signals a 'stop' in the translation of the messenger RNA. In a nonsense mutation, the genetic defect (mutation) alters a normal codon to a (premature) 'stop' codon (eg, $\underline{\mathrm{CGA}}>\underline{\mathrm{T} G A}$ ) leading to a nonfunctional protein. In the process of burning a music $C D$, there is a lead-in and lead-out region that lets the CD player know where the music begins and ends. A defect in the lead-in area may mean that the music will never be heard because the player does not know where to find the musical data. Another consequence could be a jammed CD that stops rotating in the player.

Insertions or deletions can also occur when extra base pairs are added into or removed from the DNA of a gene. This can result in the reading frame of the coding DNA going out of phase, and almost invariably results in a defective protein. In burning a music $\mathrm{CD}$, a defect in the copying process can result in the same small clip of music being played over and over again.

Finally, it is important to recognize that copying of copyright protected digital information is not permitted and yet it is extremely common. This is also the case with genetic mutations and polymorphisms throughout the human genome.

\section{ADDITIONAL READING}

1. Drayna D. Founder mutations. Sci Am 2005;293:78-85.

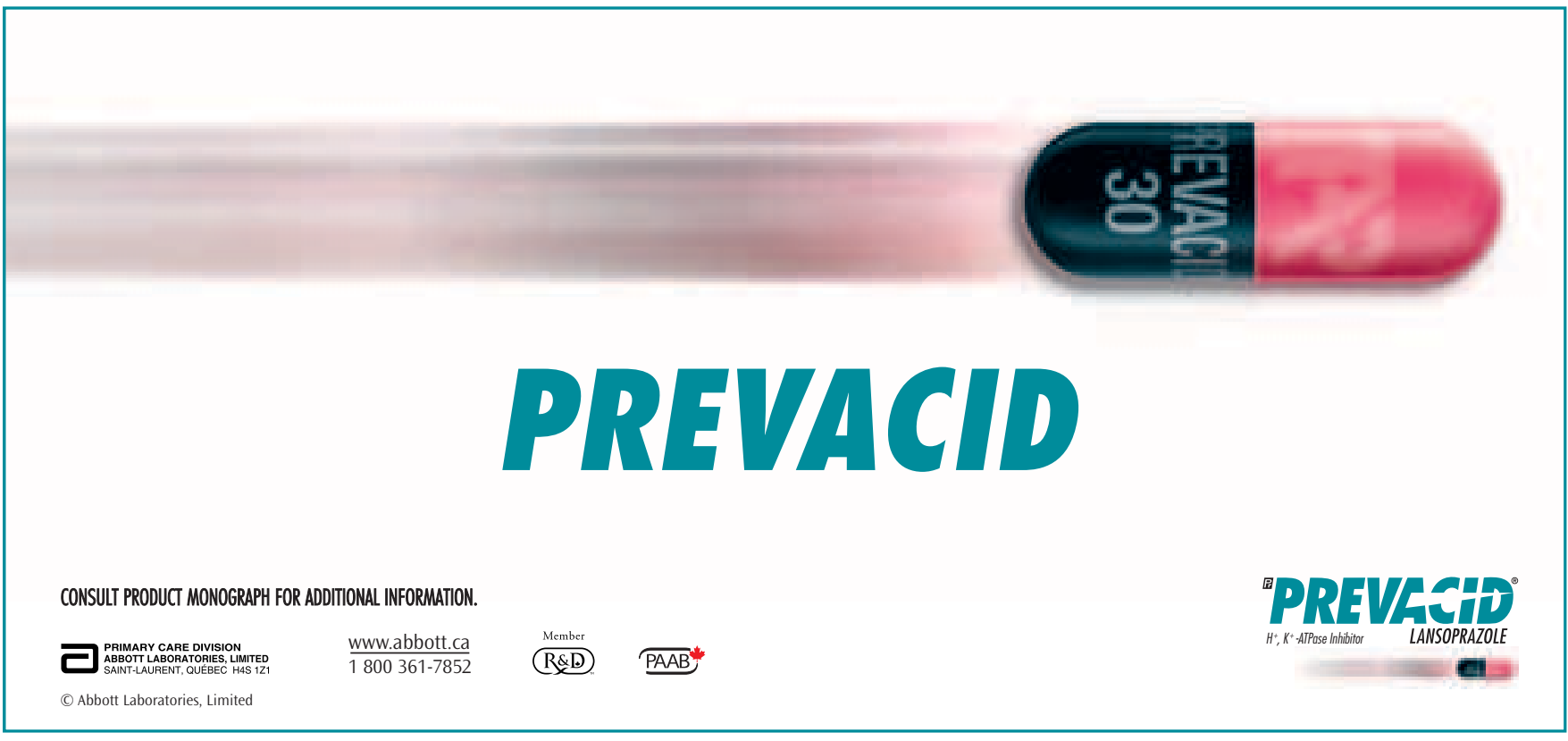




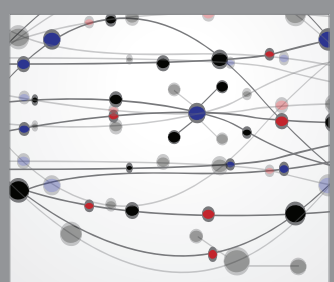

The Scientific World Journal
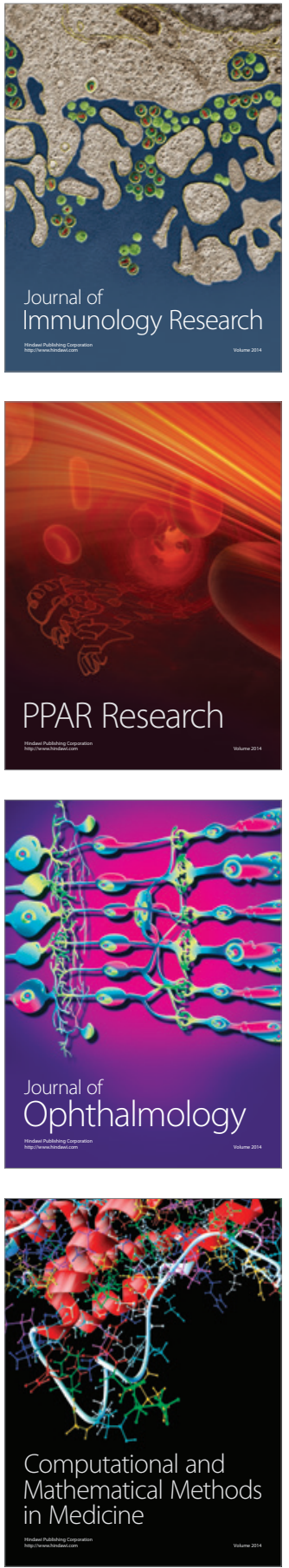

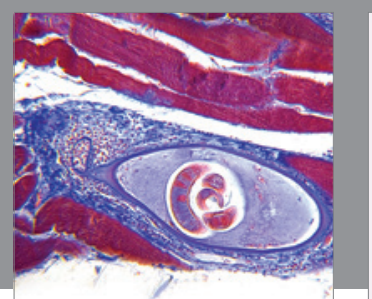

Gastroenterology Research and Practice

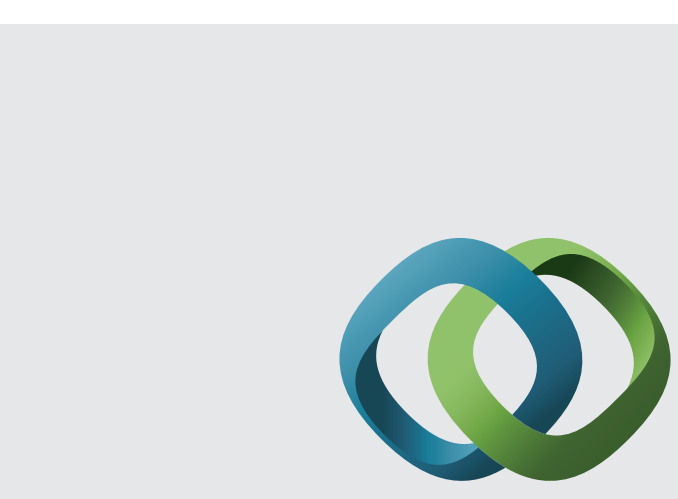

\section{Hindawi}

Submit your manuscripts at

http://www.hindawi.com
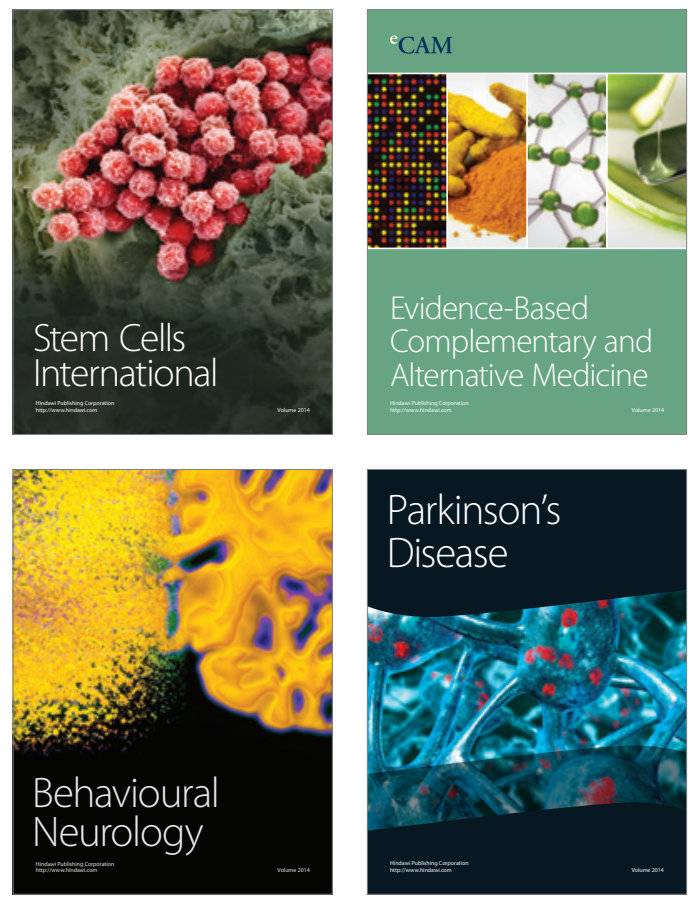
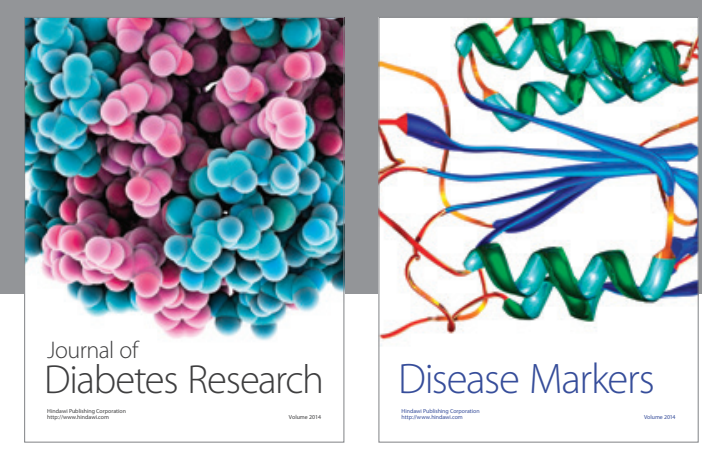

Disease Markers
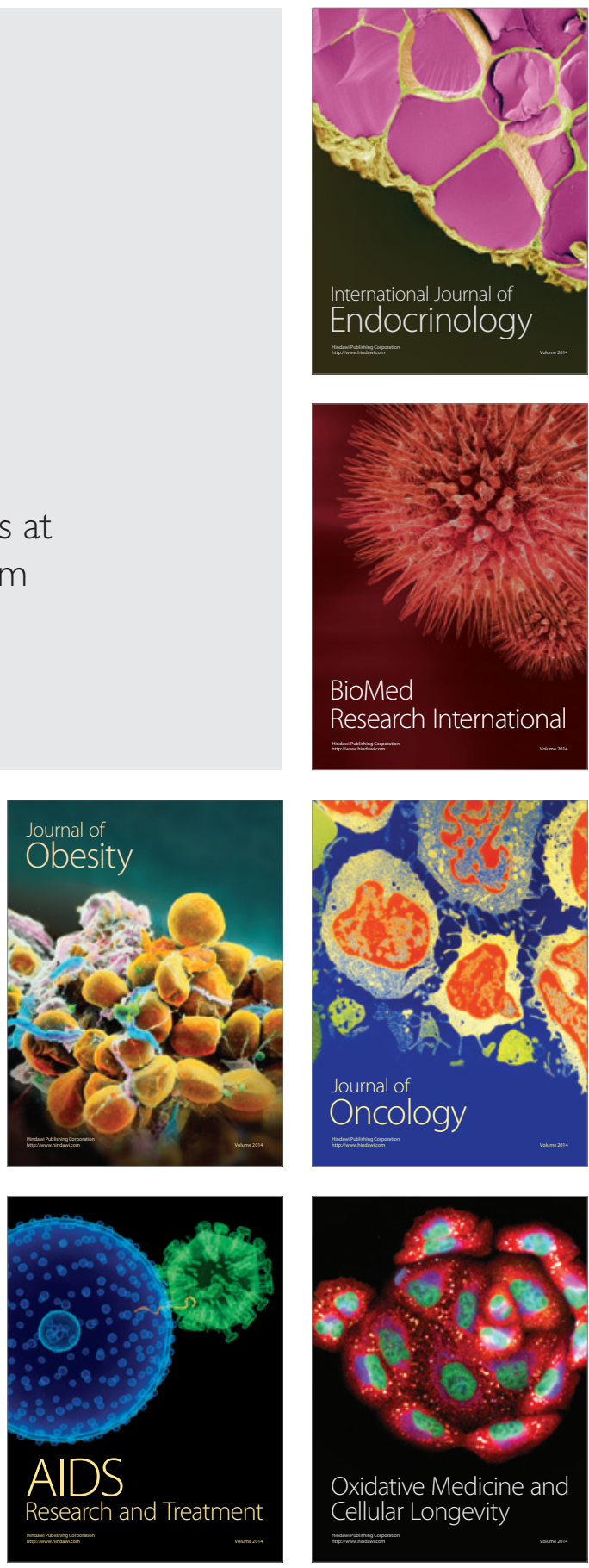